\title{
First principles calculation of isolated intermediate bands formation in a transition metal-doped chalcopyrite-type semiconductor
}

\author{
P. Palacios , K. Sánchez, J. C. Conesa, and P. Wahnón
}

Density Functional Theory (DFT) calculations at the GGA level have been carried out for Ti-substituted chalcopyritetype $\mathrm{CuGaS}_{2}$, as it might constitute an intermediate band material of the kind that has been proposed to lead to enhanced efficiency photovoltaic cells. According to these calculations an intermediate band appears when Ti substitutes Ga at a $25 \%$ level in this structure, resulting in a magnetic half- metallic compound. This intermediate band slightly overlaps the conduction band and, when a higher accuracy calculation approach like the introduction of a Hubbard-type empirical correction is used (GGA+U method), it splits leaving a filled narrow band, well isolated inside the band gap. Considering the nanocrystalline form in which these chalcopyrite-type compounds are used in solar cells, an assessment of the effects of a small crystal size in this system have been carried out with a slab model. In this calculation a decreased bandgap width is observed, which can be as a result of surface termination effects.

\section{Introduction}

In the quest for more efficient photovoltaics, a new proposal was made recently [1] in which a partially filled band is introduced inside the bandgap of a semiconductor so that the promotion of an electron from the valence band to the conduction band may be produced not only with a photon of bandgap energy or higher, but also by absorbing two photons of lower energy (Fig. 1); this leads to an increase in photocurrent without decreasing the photovoltage. A maximum ideal efficiency of $63.1 \%$ can thus be attained, much higher than the well-known Queisser limit of (single junction) normal cells $(40.7 \%)$. There is thus interest in finding material compositions able to produce the desired electronic structure. In previous works, Ti-doped GaAs and GaP were found to give the desired features [2].

In the present study another alternative is explored, carrying out such a substitution in the chalcopyrite-type semiconductor $\mathrm{CuGaS}_{2}$. Chalcopyrites form the basis for one of the most studied kinds of solar cells; in these, the semiconductor is in a polycrystalline state with a limited nanocrystal size which might influence the electronic structure and characteristics of the I.B. material. An assessment of these effects is also made here by studying thin slab-like (2D) models. 


\section{Models and methods}

The first calculations have been carried out on a model structure consisting of a supercell in which the primitive vectors are derived from the vectors of the parent body-centred tetragonal $\mathrm{CuGaS}_{2}$ structure by the equations $\mathrm{a}^{\prime}=(\mathrm{a}+\mathrm{b}+\mathrm{c}) / 2$, $b^{\prime}=(a+b-c) / 2, c^{\prime}=(b-a)$. In this cell one of the Ga atoms is then substituted by a Ti atom. This gives a centered lattice of monoclinic $\mathrm{C} 2$ symmetry, detailed in Fig. $2 \mathrm{~A}$, in which the contents of the primitive cell is $\mathrm{Cu}_{4} \mathrm{TiGa}_{3} \mathrm{~S}_{8}$ and the minimum Ti-Ti distance is ca. $6.6 \AA$.

A slab model of $\mathrm{CuGaS}_{2}$ with partial substitution of $\mathrm{Ga}$ by Ti was also constructed. The slab plane was chosen parallel to the (001) plane of the said $\mathrm{C} 2$ lattice (corresponding to the (110) plane of the parent chalcopyrite lattice); since each such plane in this lattice has a stoichiometry composition $\mathrm{CuMS}_{2}(\mathrm{M}=\mathrm{Ga}$ or $\mathrm{Ti})$, and $\mathrm{Ti}$ can be considered formally as $\mathrm{Ti}^{3+}$ species, it is electrically neutral and has no net dipole normal to it; the surface should therefore be stable enough [3]. A 7layer slab, with a thickness of ca. $12 \AA$, was chosen, and in it Ga was substituted by Ti in subsurface sites (providing the same shortest Ti-Ti distances as in the $\mathrm{Cu}_{4} \mathrm{TiGa}_{3} \mathrm{~S}_{8}$ structure) to give a composition $\mathrm{Cu}_{7} \mathrm{Ti}_{2} \mathrm{Ga}_{5} \mathrm{~S}_{14}$ and a final structure as shown in Fig. 2B. Two models with inter-slab empty spaces of ca. $18 \AA$ and $31 \AA$ in width were studied.

DFT calculations within the GGA approach (using the PW91 functional [4]) and eventually including the Hubbard correction term U [5] were carried out on the primitive cells of these structures with the VASP code [6], using a plane wave basis set with a $280 \mathrm{eV}$ energy cut-off and PAW potentials [7]. No symmetry was used in these calculations. The Brillouin zone cells were sampled according to the Monkhorst-Pack scheme; the grids used were $6 \times 6 \times 6$ (112 BZ kpoints) for the $\mathrm{Cu}_{4} \mathrm{TiGa}_{3} \mathrm{~S}_{8}$ structure, and $4 \times 4 \times 4$ ( 36 k-points) for both slab models.

Complete structure relaxation (both in atomic positions and cell sizes) was always carried out, convergence tolerances being $0.01 \mathrm{eV} / \AA$ for forces and $10^{-5} \mathrm{eV}$ for the SCF energy cycle. The PDOS in these systems has been determined by projecting the DOS within spheres with the following atomic radii for each species: 1.27, 1.47, 1.28 and $1.41 \AA$ for S, Ti, $\mathrm{Cu}$ and $\mathrm{Ga}$ respectively. In some cases calculations were carried out including both spin polarisation and mean-field Hubbard-like terms (GGA+U approach); the effective on-site Coulomb and exchange parameters used for them were $\mathrm{U}=2 \mathrm{eV}, \mathrm{J}=0.2 \mathrm{eV}$ for $\mathrm{Ti} 3 \mathrm{~d}$ electrons [8] and $\mathrm{U}=7.5 \mathrm{eV}, \mathrm{J}=0.9 \mathrm{eV}$ for $\mathrm{Cu} 3 \mathrm{~d}$ electrons [9].

\section{Results and discussion}

\section{$\underline{3.1 \text { Parent } \mathrm{CuGaS}_{2} \text { structure }}$}

The electronic structure results obtained for the parent $\mathrm{CuGaS}_{2}$ compound are summarized in the DOS data included in Fig. 3. A bandgap width of $1 \mathrm{eV}$ is observed; this is lower, as expected for a LDA/GGA result, than the value

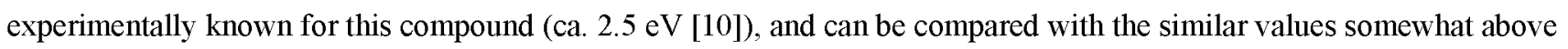
$1 \mathrm{eV}$ obtained in earlier literature works [11]. As shown by the atom-projected DOS curves the main contribution to the valence band orbitals is copper, while gallium and sulphur are the main contributors to the lower conduction band levels. Results after geometry relaxation show, compared with experimental data, an increase in cell volume of $2.89 \%$, resulting from length variations of $1.18 \%$ (on average) and $0.53 \%$ respectively along the a- and c-axes of the parent chalcopyrite structure. 


\section{$\underline{3.2 \text { Ti-substituted } \mathrm{CuGaS}_{2} \text { structure }}$}

Results for the Ti-substituted compound were obtained after geometry relaxation. The cell dimensions showed an increase in cell volume of $-0.61 \%$, resulting from length variations of $-1.11 \%$ (on average) and $+1.61 \%$ respectively along the a- and c-axes of the parent chalcopyrite structure. Ti-S bond distances of $2.35 \pm 0.01 \AA$ were found, which can be compared with the $2.31 \AA$ distance obtained for the Ga-S bond in the parent compound. Thus the decrease in cell volume can be related to the different reorganization of bond lengths in the structure. It is also noted that after relaxation (which was carried out with no symmetry constraints) the local geometry around the Ti distorts only slightly from the initial tetrahedral one: a spread of $0.05 \AA$ and $0.36^{\circ}$ in bonds and angles respectively was found around it.

Regarding the electronic structure, the DOS curves obtained from the calculation are also shown in Fig. 3. The first observation is that in these results the structure is strongly spin-polarized. Also, a distinct new feature (a relatively narrow twin-peaked band), located immediately below a bunch of states clearly identifiable with the conduction band of $\mathrm{CuGaS}_{2}$, appears in the majority spin curve. As shown by the atom-projected DOS curves, this arises almost exclusively from titanium-centered orbitals; in particular, from those of d-type. Particularly interesting is the observation that the Fermi energy crosses the afore-mentioned narrow band, and that only majority spin components appear at this energy; the corresponding minority spin ones, identifiable with the Ti-projected curve, are located significantly higher in energy, overlapping the states which correspond to the conduction band in the Ti-free parent compound.

Band diagram curves (Fig. 4) indicate that two levels contribute to this intermediate band (I.B.) feature. This was expected from the projected DOS (Fig. 3B), since in this compound the valence d-orbitals of tetrahedrally coordinated titanium will be split into two manifolds, a lower one with two levels and an upper one with three levels (these would be of e and t character respectively if the local symmetry were perfectly tetrahedral). The energy difference between the lower levels is small and probably corresponds to the aforementioned small distortion of the Ti local environment, which splits the e-type levels locally. Here titanium substitutes trivalent gallium, can be considered to be (formally) a $\mathrm{Ti}^{3+}$ species, with a $3 \mathrm{~d}^{1}$ configuration; this single electron present in the $\mathrm{d}$ shell will be then located in the lower manifold, which is thus partially filled and indeed the Fermi level is contained in the corresponding band. A distinct splitting of this band into two subbands, full and empty respectively, is not predicted by this GGA calculation.

A relevant issue, for the purposes of this work, is the location of the partially filled I.B. with respect to the other ones. In Figs. 3 and 4 it appears to overlap the conduction band-type states slightly. However, it is known that the standard DFT, which does not suppress the electronic self-interaction energy correctly, predicts empty states with energies closer to the filled ones than in the real solid, leading to too small bandgaps as found here. This defect can be corrected to a large extent using more accurate (and computer time-consuming) methods as exact exchange (EXX) or GW [12], and it is very likely that if any such method were used one would find the conduction band at a higher energy, and at a narrower I.B. (since the complete correction of the self-interaction will allow the d-states to be more localized with less overlapping), leading to a clear separation between the I.B. and the conduction band. This was indeed seen to occur when applying the EXX calculation method to the related Ti:GaP system [13]. Thus $\mathrm{CuGaS}_{2}$ is a good candidate to make up an I.B. material, with enhanced photovoltaic properties, when Ti substitutes Ga in it.

These results for this material also indicate possible half-metallic magnetic properties, which could also imply interesting applications in spintronics as in the case of Mn-substituted III-V compounds [14]. Since these features depend strongly on the degree of electronic correlation, for a better description GGA+U calculations were carried out on jit. Our results (Fig. 5) show a full splitting of the e -type levels, that of the lower majority-spin being fully occupied, and no electron 
density at the Fermi level. Thus a half-metallic character may after all be absent here; a detailed examination of this issue, where the effect of $\mathrm{Ti}$ concentration may be determinant, is under way. A significant increase in comparison with the GGA results is seen, as well as, in the contribution of sulphur to the valence band.

\section{$\underline{3.3 \text { Ti-substituted } \mathrm{CuGaS}_{2} \text { slab }}$}

Considering that chalcopyrite-type semiconductors similar to $\mathrm{CuGaS}_{2}$, when used in photovoltaic cells, are grown as polycrystalline thin films, it was considered convenient to study the possible effect of small crystal size on the I.B. characteristics. For this purpose a 2-dimensional slab model as described above was built, its cell dimensions and atomic positions were relaxed to obtain the minimum energy, and the resulting electronic structure was analyzed. The atom position changes were now larger than for the 3D periodic solid, mainly as a result of movements of the outer surface atoms trying to compensate for the missing bond interactions; but no new bond seemed to appear in this relaxed configuration, indicating that dangling bond effects were not very important. Rather, the exposed $\mathrm{Ga}$ and $\mathrm{Cu}$ atoms relaxed inwards to achieve a near planar trigonal coordination while sulphur atoms, also 3-fold coordinated, remained exposed in a pyramidal configuration (since Ti was not exposed in this model, no relevant coordination change appeared in it). This is consistent with a significantly ionic description of the material so that, although sulphur behaves as covalently bonded, with a coordination geometry indicating the effect of a lone $\mathrm{sp}^{3}$ pair, the $\mathrm{Cu}$ and $\mathrm{Ga}$ atoms behave as closed shell spherical ions around which sulphur ligands locate so as to minimize the mutual repulsions.

Figure 6 presents the DOS results. The PDOS data displayed in it are those found for the model with an $18 \AA$ thick empty space; those for the model with larger empty space are rather similar (see the comparison of total DOS in Fig. 6A), the small differences appearing probably resulting from residual electrostatic interaction between the slabs' quadrupolar moments (their dipolar moments are 0). The data show that the spin-polarized I.B. electronic structure is qualitatively preserved, but some differences appear in comparison to the bulk system (Fig. 3): the gap between the main valence band at $\mathrm{E}<-1 \mathrm{eV}$ and the Ti states below the Fermi level shows a small reduction (maybe because of the destabilization of the surface atoms' electron density as a result of the coordination decrease), and the splitting of the Ti $3 \mathrm{~d}$ features in two components becomes clearer. The structure of these Ti states is otherwise relatively unaffected; this is probably because these atoms were placed in subsurface positions within the model used, so that the main interactions affecting them were not significantly influenced by the termination of the lattice, and indeed the average Ti-S bond length $(2.37 \AA)$ is quite similar to that obtained for the bulk compound.

As said above, a more accurate assessment of the real existence of an overlap between the I.B. and the conduction band to ascertain whether this nanostructured material would be valid for building an I.B. photovoltaic cell would require using more advanced theoretical methods such as EXX or GW, involving computationally very expensive work that has not been attempted yet here.

\subsection{Thermodynamic viability}

A first assessment of the possibility of obtaining experimentally such Ti-substituted chalcopyrite can be made evaluating the energetics of its decomposition in known stable compounds. In principle, one could consider the decomposition into $\mathrm{CuGaS}_{2}$ and $\mathrm{CuTiS}_{2}$. However, no reports of a $\mathrm{CuTiS}_{2}$ compound could be found in the literature. The closest one is $\mathrm{Cu}_{0.6} \mathrm{TiS}_{2}$ [15], with a structure derived from the layered one of TiS2 by disordered intercalation of $\mathrm{Cu}$ atoms between the 
layers so as to achieve a tetrahedral coordination for them. This results in a compound with (formally) copper in a $\mathrm{Cu}^{+}$ state and titanium in a mixed valence $\mathrm{Ti}^{4+} / \mathrm{Ti}^{3+}$ state. Using a correspondingly ordered $\mathrm{Cu}_{23} \mathrm{TiS}_{2}$ structure as the closest model, the following model for a likely decomposition can be presumed:

$\mathrm{Cu}_{4} \mathrm{TiGa}_{3} \mathrm{~S}_{8} \rightarrow 3 \mathrm{CuGaS}_{2}+\mathrm{Cu}_{2 / 3} \mathrm{TiS}_{2}+1 / 3 \mathrm{Cu}$

A preliminary evaluation of the energetics of this process, through similar DFT methods as used here, yields an energy balance of $\Delta \mathrm{E}=-0.75 \mathrm{eV}$ for this process. Thus Ti-substituted $\mathrm{CuGaS}_{2}$ would be unstable against decomposition. This is probably related to the fact that in the chalcopyrite structure $\mathrm{Ti}$ would have a tetrahedral coordination rather than the 6fold one that this element prefers and is present in the known $\mathrm{Cu}$, Ti sulphide. However, as discussed in a recent work carried out on the related $\mathrm{Ti}_{y} \mathrm{Ga}_{1-y} \mathrm{X}$ system [16], in which the corresponding energy balance was found to be almost twice as high, this does not mean that the insertion of $\mathrm{Ti}$ in the tetrahedral semiconductor is unfeasible, since the analogous system of $\mathrm{Mn}$ insertion in GaAs, which has been proven experimentally to be possible for Mn concentrations of up to ca. $5 \%$, yields an unfavourable transition metal insertion energy of up to $2.0 \mathrm{eV}$ in a similar calculation. Thus one can expect that making this Ti-substituted chalcopyrite material is practically possible.

\section{Conclusions}

The electronic structures computed for $\mathrm{CuGaS}_{2}$-derived structures in which $\mathrm{Ga}$ is partially substituted by titanium show that these compounds might be a basis for obtaining intermediate band materials suitable for recently proposed highperformance photovoltaic cells. These electronic structures are consistent with the e-like levels, containing one electron per inserted atom, that are expected to appear upon this $\mathrm{Ti}$ substitution in these tetrahedral semiconductors. If these materials are made into mono- or bi- dimensional nanostructures, surface effects leading to effective bandgap narrowing may occur. Ways to avoid this undesired effect should be devised, e.g. by increasing the crystallite size or passivating the surfaces with atoms able to compensate for the decreased bonding produced by the lattice termination.

Acknowledgements We acknowledge funding from project FULLSPECTRUM (contract SES-CT-2003-502620) of the $6^{\text {th }}$ European Framework Programme and from the Spanish National R\&D contract MAT2004-04631.

\section{$\underline{\text { References }}$}

[1] A. Luque and A. Martí, Phys. Rev. Lett. 78, 5014 (1997).

[2] P. Wahnón and C. Tablero, Phys. Rev. B 65, 165115 (2002); P. Wahnón, P. Palacios, J. J. Fernández, and C. Tablero, J. Mater. Sci. 40, 1383 (2005).

[3] F. Bertaut, Comp. Rend. Acad. Sci. 246, 3447 (1958).

[4] J. P. Perdew and Y. Wang, Phys. Rev. B 45, 13244 (1992).

[5] S. L. Dudarev, G. A. Botton, S. Y. Savrasov, C. J. Humphreys, and A. P. Sutton, Phys. Rev. B 57, 1505 (1998).

[6] G. Kresse and J. Hafner, Phys. Rev. B 47, RC558 (1993); G. Kresse and J. Furthmüller, Phys. Rev. B 54, 11169 
(1996).

[7] G. Kresse and J. Joubert, Phys. Rev. B 59, 1758 (1999).

[8] A. I. Poteryaev, A. I. Lichenstein, and G. Kotliar, Phys. Rev. Lett. 93, 086401 (2004).

[9] A. I. Liechtenstein, V. I. Anisimov, and J. Zaanen, Phys. Rev. B 52, R5467 (1995).

[10] S. Shirakata, K. Murakami, and H. Kukimoto, Jpn. J. Appl. Phys. 28(1), 1728 (1989).

[11] S. Picozzi, Y. J. Zhao, A. J. Freeman, and B. Delley, Phys. Rev. B 66, 205206 (2002); J. E. Jaffe and A. Zunger, Phys. Rev. B 28, 5822 (1983).

[12] M. Städele, M. Moukara, J. A. Majewski, P. Vogl, and A. Görling, Phys. Rev. B 59, 10031 (1999).

[13] J. J. Fernández, C. Tablero, and P. Wahnón, J. Chem. Phys. 120, 10780 (2004).

[14] L. Kronik, M. Jain, and J. R. Chelikowsky, Phys. Rev. B 66, 041203 (2002); M. Wierzbowska, D. Sánchez Portal, and S. Sanvito, Phys. Rev. B 70, 235209 (2004); S. Sanvito, G. Theurich, and N. A. Hill, J. Supercond. Novel Magn. Mater. 15, 85 (2002); S. Sanvito, P. Ordejón, and N. A. Hill, Phys. Rev. B 63, 165206 (2001)

[15] N. Le Nagard, O. Gorochov, and G. Collin, Mat. Res. Bull. 10, 1287 (1975).

[16] P. Palacios, P. Wahnón, S. Pizzinato, and J. C. Conesa, J. Chem. Phys. 124, 014711 (2006). 


\section{Captions of figures}

Fig. 1 Scheme of an I.B. photovoltaic cell.

Fig. 2 Studied structures: A) $\mathrm{Cu}_{4} \mathrm{TiGa}_{3} \mathrm{~S}_{8}$ centered monoclinic cell; B) $\mathrm{Cu}_{7} \mathrm{Ti}_{2} \mathrm{Ga}_{5} \mathrm{~S}_{14}$ slab (4 primitive cells are shown). Color coding: white $=\mathrm{S}$; black $=\mathrm{Ti}$; light orange $=\mathrm{Cu}$; dark green $=\mathrm{Ga}$.

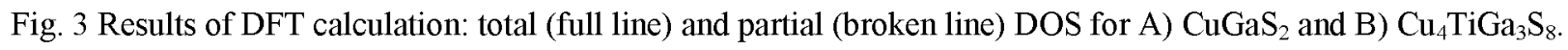

Fig. 4 Selected parts of $\mathrm{Cu}_{4} \mathrm{TiGa}_{3} \mathrm{~S}_{8}$ band diagram for majority (left side) and minority (right side) spin.

Fig. 5 Results of GGA+U calculations for $\mathrm{Cu}_{4} \mathrm{TiGa}_{3} \mathrm{~S}_{8}$ : A) Total DOS and B) Partial (projected) DOS.

Fig. 6 A) Total DOS for the $\mathrm{Cu}_{7} \mathrm{Ti}_{2} \mathrm{Ga}_{5} \mathrm{~S}_{14}$ slab with inter-slab empty spaces of $18 \AA$ (in red) and $31 \AA$ (in green), and B) partial DOS for the $\mathrm{Cu}_{7} \mathrm{Ti}_{2} \mathrm{Ga}_{5} \mathrm{~S}_{14}$ slab with a $18 \AA$ inter-slab empty space. 
Fig. 1

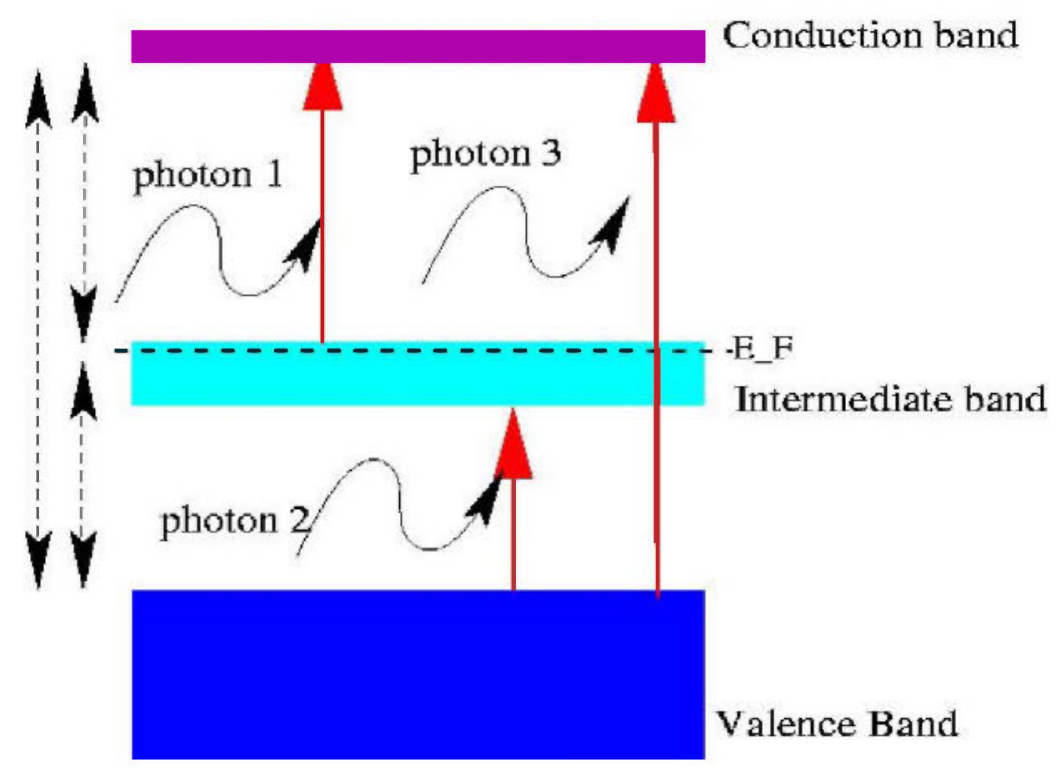


Fig. 2

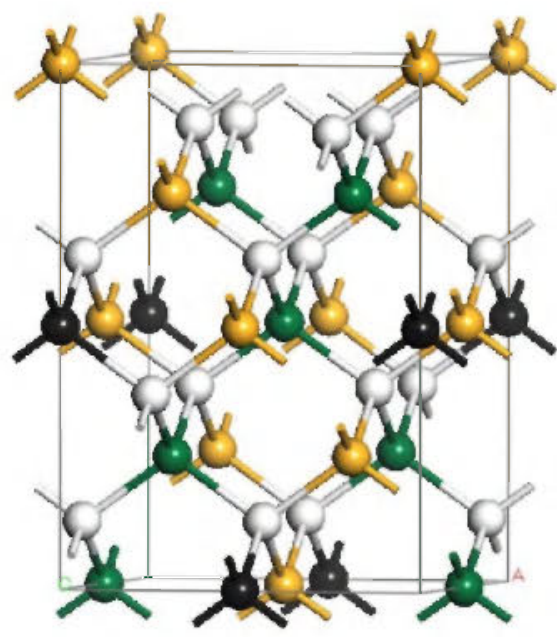

A)

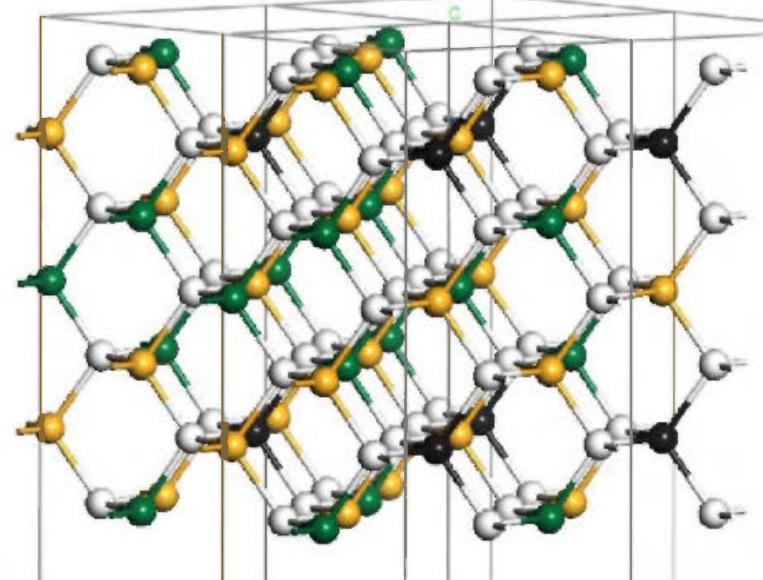

B) 
Fig. 3
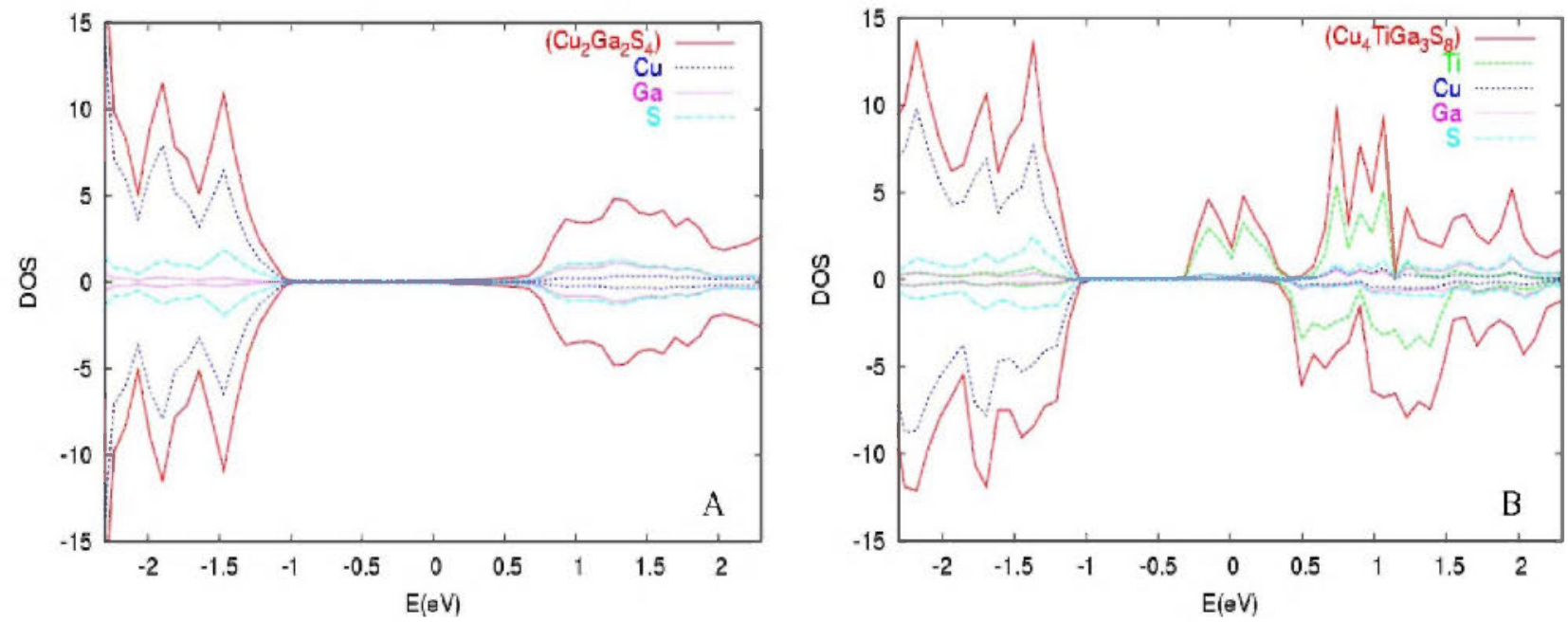
Fig. 4

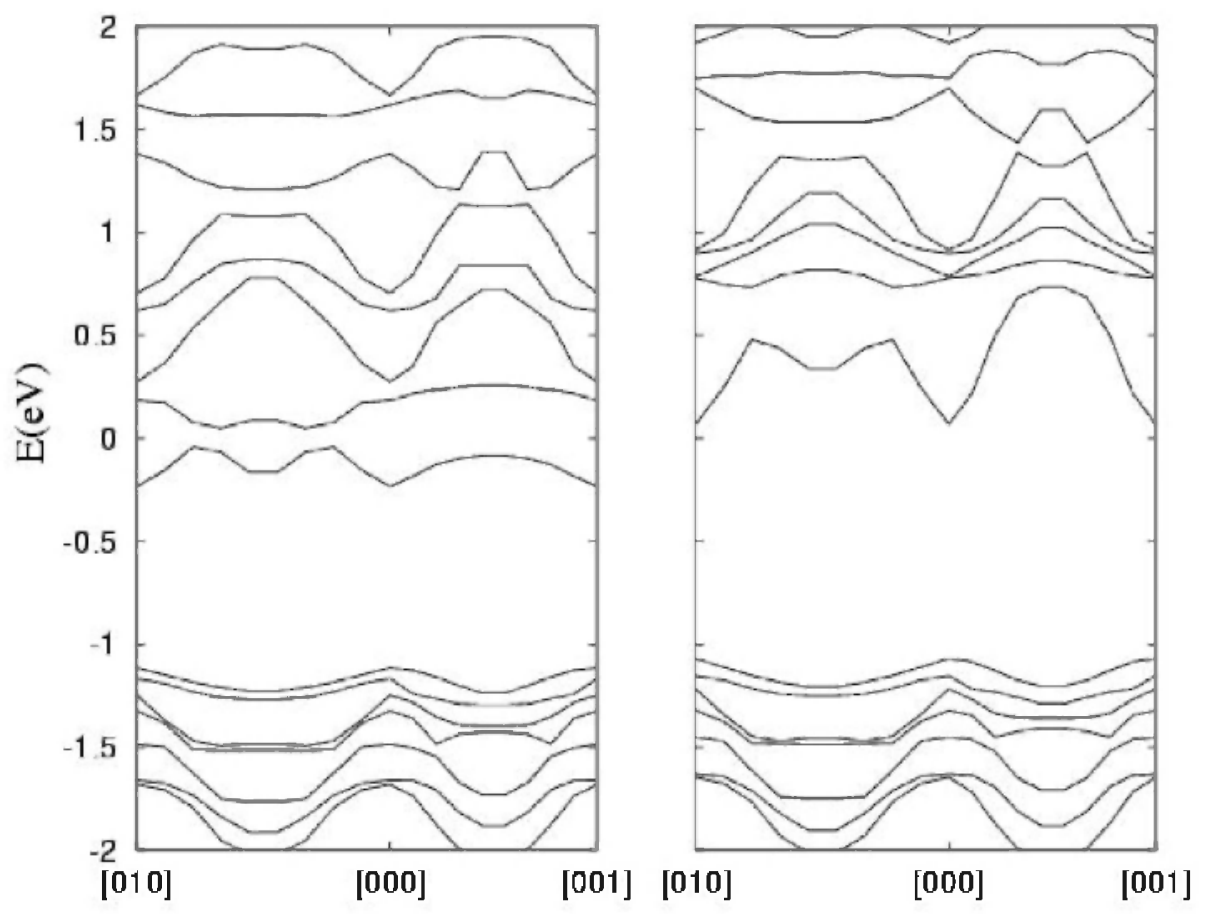


Fig. 5
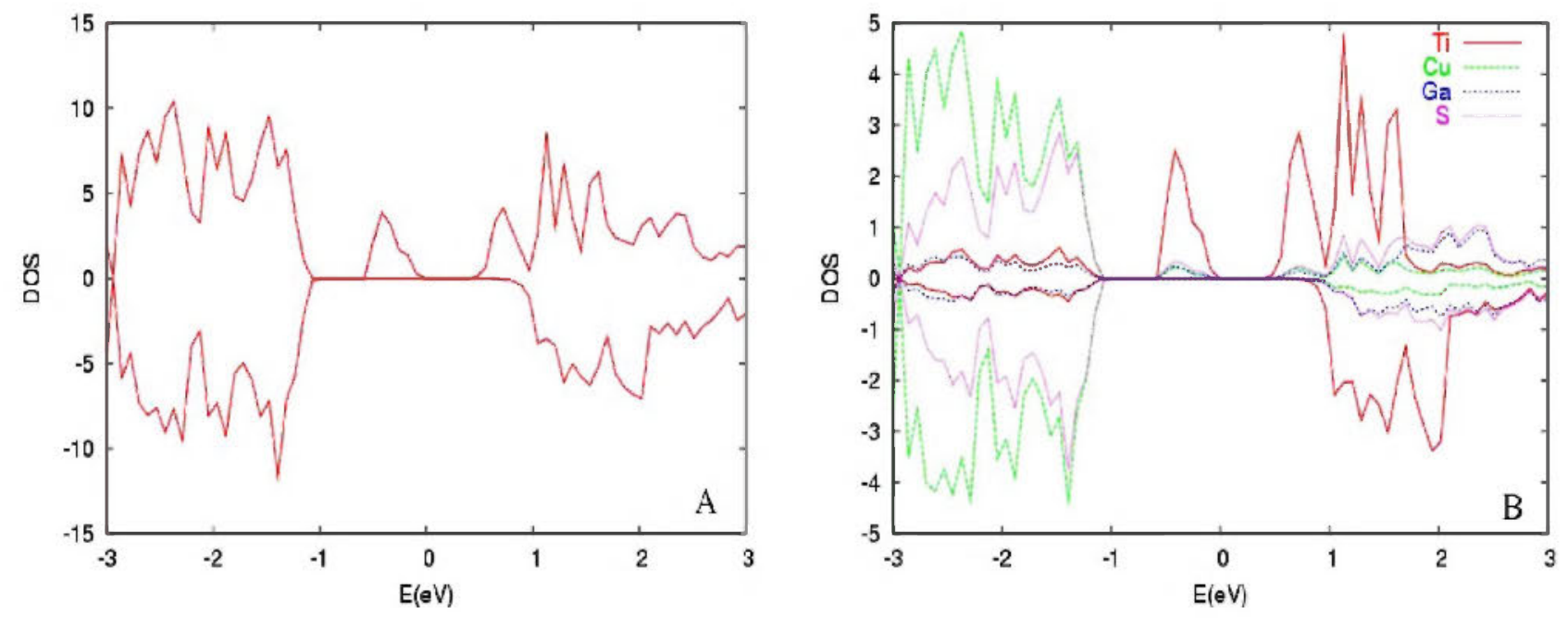
Fig. 6
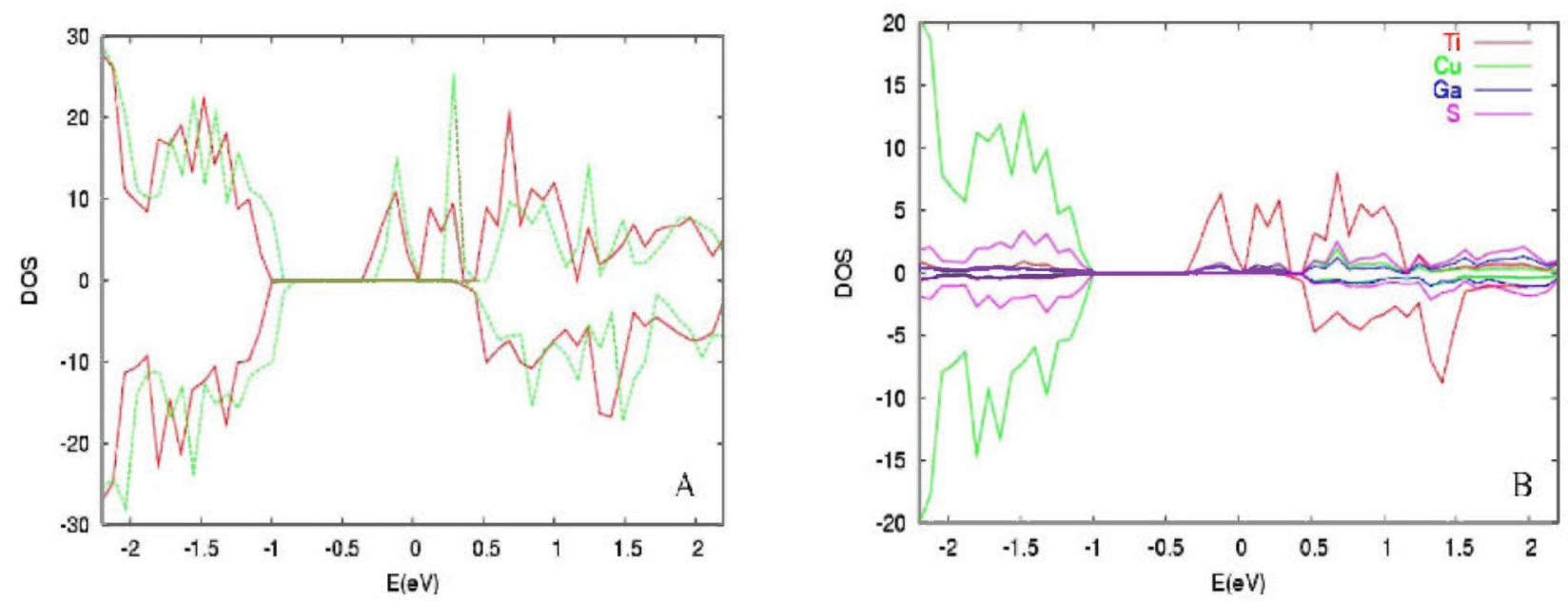